\title{
Data-Driven Subgroups in Depression Derived from Directed Functional Connectivity Paths at Rest
}

\author{
Rebecca B Price ${ }^{*, 1}$, Kathleen Gates ${ }^{2}$, Thomas E Kraynak ${ }^{3}$, Michael E Thase ${ }^{4}$ and Greg J Siegle' \\ 'Department of Psychiatry, University of Pittsburgh, Pittsburgh, PA, USA; 'University of North Carolina-Chapel Hill, Chapel Hill, NC, USA; \\ ${ }^{3}$ Department of Psychology, University of Pittsburgh, Pittsburgh, PA, USA; ${ }^{4}$ Perelman School of Medicine of the University of Pennsylvania and the \\ Philadelphia Veterans Affairs Medical Center
}

\begin{abstract}
Depressed patients show abnormalities in brain connectivity at rest, including hyperconnectivity within the default mode network (DMN). However, there is well-known heterogeneity in the clinical presentation of depression that is overlooked when averaging connectivity data. We used data-driven parsing of neural connectivity to reveal subgroups among 80 depressed patients completing resting state $f M R I$. Directed functional connectivity paths (eg, region A influences region B) within a depression-relevant network were characterized using Group Iterative Multiple Model Estimation, a method shown to accurately recover the direction and presence of connectivity paths in individual participants. Individuals were clustered using community detection on neural connectivity estimates. Subgroups were compared on network features and on clinical and biological/demographic characteristics that influence depression prognosis. Two subgroups emerged. Subgroup A, containing 7I\% of the patients, showed a typical pattern of connectivity across DMN nodes, as previously reported in depressed patients on average. Subgroup B exhibited an atypical connectivity profile lacking DMN connectivity, with increased dorsal anterior cingulate-driven connectivity paths. Subgroup B members had an over-representation of females ( $87 \%$ of Subgroup B vs $65 \%$ of Subgroup $A ; \chi^{2}=3.89, p=0.049$ ), comorbid anxiety diagnoses (42.9\% of Subgroup B vs 17.5\% of Subgroup $A ; \chi^{2}=5.34, p=.02$ ), and highly recurrent depression (63.2\% of Subgroup B vs $31.8 \%$ of Subgroup $A ; \chi^{2}=5.38, p=.02$ ). Neural connectivity-based categorization revealed an atypical pattern of connectivity in a depressed patient subset that would be overlooked in group comparisons of depressed and healthy participants, and tracks with clinically relevant phenotypes including anxious depression and episodic recurrence. Data-driven parsing suggests heterogeneous substrates of depression; ideally, future work building on these findings will inform personalized treatment. Neuropsychopharmacology (2017) 42, 2623-2632; doi: I 0.1038/npp.2017.97; published online 21 June 2017
\end{abstract}

\section{INTRODUCTION}

There is substantial heterogeneity in the clinical presentation of depression. In a representative treatment-seeking sample of 3703 depressed patients, over 1000 unique symptom profiles were observed (Fried and Nesse, 2014). Thus, although group comparisons of depressed and healthy samples have revealed numerous biological and neural features that track with depression on the whole, group averages mask considerable heterogeneity and may not accurately represent even a single individual patient (Gates and Molenaar, 2012; Miller et al, 2002; Molenaar and Campbell, 2009). We (Price et al, 2017) and others (Clementz et al, 2016; Drysdale et al, 2017; Karalunas et al, 2014; Yang et al, 2014) have reported that data-driven parsing of biobehavioral heterogeneity within disorder domains (eg, attention deficit, psychosis, and depression) can yield biologically based subgroups that predict external measures

\footnotetext{
*Correspondence: Dr RB Price, Department of Psychiatry, Western Psychiatric Institute and Clinic, University of Pittsburgh, 381 I O'Hara Street, Pittsburgh, PA 15213, USA, Tel: + 412383 5443, Fax: + 412 383 4077, E-mail: rebecca.price@stanfordalumni.org

Received 9 January 2017; revised 7 April 2017; accepted 5 May 2017; accepted article preview online 12 May 2017
}

of functioning, clinical outcomes, and neurobiology with better precision than traditional diagnostic subgroups.

Depressive heterogeneity notwithstanding, a large literature suggests depression-related alterations in neural connectivity during the resting state (RS). RS connectivity patterns tend to exhibit trait-like stability over time with a high degree of individual specificity (Finn et al, 2015) and are posited to represent neural functional architecture that remains consistent across diverse conditions (eg, under anesthesia). Meta-analyses suggest as a group, depressed individuals exhibit elevated RS connectivity within regions of the default mode network (DMN; (Kaiser et al, 2015)), a network that deactivates during many tasks and is associated with internal mentation, including self-referential processing (Andrews-Hanna et al, 2010) and negative rumination (Zhu et al, 2012). Hyperconnectivity across the DMN and regions of the cognitive control network (CCN), as well as hypoconnectivity between $\mathrm{DMN}$ and ventral affective network (VAN) regions, were also reported in a meta-analysis (Kaiser et al, 2015). However, the direction of RS findings in individual studies of depression (eg, hyper vs hypoconnectivity) is sometimes conflicting (Hasler and Northoff, 2011; Kaiser et al, 2015), even though RS methods are considered highly translatable and reliable. Conflicting findings are to be 
expected if meaningful neural heterogeneity is present within depressed patients but overlooked in conventional groupbased analysis. This may lead to mixed or spurious findings (Gates and Molenaar, 2012; Miller et al, 2002; Molenaar and Campbell, 2009), incomplete etiological models, and confusion within the literature, while overlooking biological subgroups that may represent unique etiologies requiring unique treatments. Consistent with this suggestion, a recent data-driven approach suggested as many as four subtypes of RS connectivity patterns within depression may be conflated when depressed patients are averaged together (Drysdale et al, 2017).

Clinically, one of the most widely acknowledged forms of heterogeneity within depressed patients is the presence or absence of comorbid anxiety. Roughly half of treatmentseeking depressed patients report clinically significant anxiety, which is associated with poor prognosis and treatment response (Fava et al, 2008), greater severity and disability, and higher risk of severe outcomes (eg, suicidality; (Fava et al, 2004)). Few studies have examined the neural substrates of this potential dichotomy within depressed patients. During task performance, anxious depression may have unique neural activation substrates spanning VAN, DMN, and CCN regions (Demenescu et al, 2011; Etkin and Schatzberg, 2011b; van Tol et al, 2012; van Tol et al, 2011). However, brain processes may best be characterized as the coordinated activity of disparate brain regions over time (Heller et al, 2009; Sporns et al, 2004). RS connectivity offers a glimpse at this coordinated activity in the absence of task demands, which may capture idiosyncratic, endogenous, habitual processing patterns akin to the habitual and intrusive anxious thought patterns and affective states reported by patients. In studies comparing RS connectivity patterns for depressed individuals with and without comorbid anxiety (assessed both categorically and continuously), unique patterns between and within the VAN, DMN, and CCN have been reported (Oathes et al, 2015; Pannekoek et al, 2015). Findings are generally consistent with the view that anxiety and depression have both common and unique neural substrates, informing an important etiological debate by supporting both 'shared diathesis' and 'independentfactor' models of psychopathology (Etkin and Schatzberg, 2011b). However, the patterns detectable within the neural data in previous studies are necessarily constrained by the investigators' selection of independent variables (ie, anxiety measures).

A more novel approach, facilitated by recent advances in data-driven clustering, is to begin by parsing the heterogeneity contained in neural connectivity maps themselves, enabling detection of biologically derived subgroups with unique neural connectivity profiles. This approach allows the optimal number of subgroups (from one-implying homogeneity-to the full sample size-implying no meaningful similarities) to emerge organically from the connectivity data. Subgroups can then be independently characterized with respect to clinically relevant, observable characteristics. We have previously used this approach in conjunction with a connectivity method-Group Iterative Multiple Model Estimation (GIMME (Gates and Molenaar, 2012; Gates et al, in press))-shown to reliably recover, for each individual, both the presence and the direction of connectivity among regions (ie, does A predict B after controlling all other network-wide influences (including B's influence on itself?)). GIMME was motivated by a seminal paper that found no methods to date could reliably detect individual-level models in simulated data (Smith et al, 2011). These simulations reflected common issues seen in fMRI data such as lower signal-to-noise ratio, nonstationarity, poor region of interest (ROI) selection, and HRF deviation within individuals, and GIMME was found to perform excellently in these cases (Gates and Molenaar, 2012). A recent independent review highlighted GIMME as one of the only approaches that can reliably capture both the presence and direction of functional connectivity paths within heterogeneous individuals (Mumford and Ramsey, 2014). Furthermore, in extensive Monte Carlo simulations, Subgroup-GIMME ('S-GIMME') can arrive at the correct cluster assignments nearly perfectly, even in sample sizes as small as 25 , and at rates far higher than clustering based on more traditional connectivity metrics such as correlation matrices (Gates et al, in press). When applied to fMRI data collected from depressed and healthy individuals during a positive mood induction, the resulting connectivity-based subgroups predicted presence or absence of depression, as well as numerous clinically relevant indices of affective dysregulation (Price et al, 2017).

Here we applied this data-driven, brain-based categorization approach to RS functional connectivity maps obtained from 80 depressed patients (overlapping with those in our previous report; see Supplementary Information), across key nodes of three networks that show well-replicated roles in depressive symptomatology: DMN, CCN, and VAN. Unique goals of the present analysis were to focus explicitly on parsing fine-grained heterogeneity within depressed patients (rather than more broadly across both depressed and healthy individuals) and to focus on the RS, which has been widely studied in depression and shows promise as a method for identifying clinically relevant biotypes (Drysdale et al, 2017). S-GIMME produces subgroup-specific network connectivity maps, informing an empirical data-driven model of connectivity subtypes within depression. Based on previous research suggesting anxious depression may represent a unique phenotype with high clinical relevance, we then assessed the external relevance of connectivity-based subgroups in predicting comorbid anxiety diagnoses. In an effort to further understand whether study-specific sample composition parameters may contribute to variable findings when simple depressed $v s$ healthy group comparisons are used, we examined several other important clinical (severity and recurrence) and biological (gender and age) features routinely reported as sample characteristics in existing studies. Our data-driven approach has the capacity to reveal heterogeneity within functional neural architecture that is masked by traditional group comparisons and ultimately could inform development of discrete treatments targeting discrete neurobiological etiologies.

\section{MATERIALS AND METHODS}

Participants were 80 unmedicated MDD patients with moderate-to-severe depression $(\mathrm{BDI}$ mean $=30.73 ; \mathrm{SD}=9.5)$ recruited for a larger treatment study (Siegle et al, 2012; Supplementary Information and Table 1). 
Table I Subgroup Clinical and Demographic/Biological Characteristics

\begin{tabular}{|c|c|c|c|c|c|}
\hline & $\begin{array}{c}\text { Subgroup A } \\
(n=57)\end{array}$ & $\begin{array}{c}\text { Subgroup B } \\
(n=23)\end{array}$ & $\begin{array}{l}\text { Statistic testing group } \\
\text { differences }\end{array}$ & $\begin{array}{c}\text { Statistical } \\
\text { significance }(p)\end{array}$ & $\begin{array}{l}\text { Effect size } \\
(95 \% \mathrm{Cl})\end{array}$ \\
\hline$\geq \mid$ Comorbid anxiety disorder & $\begin{array}{c}\text { I } 7.5 \%(n=10 / 57) \\
n=3 \text { GAD } \\
n=\mid \text { PTSD } \\
n=1 \text { Specific phobia } \\
n=7 \text { Social phobia }\end{array}$ & $\begin{array}{c}42.9 \%(n=9 / 21) \\
n=2 \text { GAD } \\
n=2 \text { PTSD } \\
n=1 \text { Specific phobia } \\
n=7 \text { Social phobia } \\
n=1 \text { Panic disorder } \\
n=2 \text { Anxiety NOS }\end{array}$ & $\chi^{2}=3.89$ & 0.021 & $O R=3.53(1.17-10.60)$ \\
\hline Severe MDD & $19.3 \%(n=11 / 57)$ & $38.1 \%(n=8 / 21)$ & $\chi^{2}=2.94$ & 0.086 & $\mathrm{OR}=2.57(0.86-7.73)$ \\
\hline $\begin{array}{l}\text { Highly recurrent MDD } \\
\text { ( } \geq 3 \text { episodes) }\end{array}$ & $31.8 \%(n=14 / 44)$ & $63.2 \%(n=12 / 19)$ & $\chi^{2}=5.38$ & 0.020 & $\mathrm{OR}=3.67(1.19-11.34)$ \\
\hline Sex (\% female) & $64.9 \%(n=37 / 57)$ & $87.0 \%(n=20 / 23)$ & $\chi^{2}=3.89$ & 0.049 & $\mathrm{OR}=3.60(1.18-11.00)$ \\
\hline Mean age (SD) & $35.3(\mid 1.1)$ & $37.3(11.6)$ & $t_{78}=.72$ & 0.473 & $d=0.18(-0.31$ to 0.66$]$ \\
\hline
\end{tabular}

Abbreviations: GAD, generalized anxiety disorder; MDD, major depressive disorder; NOS, not otherwise specified; PTSD, posttraumatic stress disorder,

Some individuals ( $n=2$ Subgroup A; $n=3$ Subgroup B) met criteria for more than one comorbid anxiety diagnosis. Decreased total N's in denominators are due to loss of diagnostic data due to database error (two participants) and insufficient information to confidently determine number of previous episodes and/or duration of current episode ( 15 participants). Bold $=p<0.05$; italics $=p<0.10$.

\section{fMRI Acquisition and Preprocessing}

Data were acquired during a 7 min eyes-open RS block. T2*weighted images depicting $\mathrm{BOLD}$ contrast $(\mathrm{TR}=1500$; $\mathrm{TE}=27 ; \quad \mathrm{FOV}=24 \mathrm{~cm}$; flip angle $=80^{\circ} ;$ Twenty-nine $3.2 \mathrm{~mm}$ slices; $280 \mathrm{TRs}$ ) were acquired on a 3T Siemens Allegra $(n=4)$ or a $3 \mathrm{~T}$ Siemens Trio $(n=76)$. Standard preprocessing steps were applied (see (Price et al, 2017; Supplementary Information). AFNI's ANATICOR algorithm was applied to remove artifacts (hardware and motion) that may influence connectivity estimates. AFNI's 3dvolreg motion correction algorithm was applied. Timepoints with incremental translational/rotational movement $\geqslant 0.5 \mathrm{~mm}$ or $0.5^{\circ}(1.7 \%$ of data) were removed from analysis (marked as missing data).

Fifteen ROIs were selected $a$ priori based on prior literature in depression (emphasizing replicated and meta-analytic findings) with the goal of spanning networks relevant to ventral affective processing (VAN), self-referential processing $(\mathrm{DMN})$, and top-down regulation (CCN). See Supplementary Information and Figure 1 for details of ROI definitions.

\section{Directed Connectivity and Community Detection}

The full sample of 80 individuals was processed and clustered without regard to clinical/demographic features. Directed paths (ie, establishing which of two ROIs statistically predicts the other after controlling for other candidate regions (including lagged auto-regressions)) between all pairs of ROIs (both contemporaneous and at lag $=1 \mathrm{TR}$ ) were derived for each individual using S-GIMME (Gates et al, in press; Lane et al, 2015). Briefly, using a unified structural equation framework (Kim et al, 2007) and a Bayes net formulation, S-GIMME first looks across individuals to detect signal from noise and arrive at a map of lagged and contemporaneous directed connections that exist for the majority ('group-level map'). Next, S-GIMME arrives at a similarity matrix using the individual-level estimates of these group-level connections, as well as anticipated estimates for candidate connections. Walktrap (Pons and Latapy, 2006), an 'unsupervised' community detection algorithm found to be robust across many issues common in clustering (eg, unequal cluster sizes; (Orman and Labatut, 2009)), is conducted on this matrix to arrive at an optimal number of subgroups who have shared connectivity patterns (similar strength, sign (positive/negative), temporal pattern (contemporaneous/ lagged), and direction (eg, region $\mathrm{A} \rightarrow$ region $\mathrm{B}$ ) of connectivity paths). S-GIMME then searches for subgrouplevel paths. Finally, S-GIMME robustly identifies individuallevel connections using group- and subgroup-derived temporal patterns as a starting point. S-GIMME thus generates group-level, subgroup-specific, and individual (per-participant) connectivity maps characterizing the network structure.

\section{External Variables}

Subgroups were independently tested and characterized across several clinical and demographic features selected a priori based on established clinical relevance and interpretability. Selected variables were those likely to be reported as sample characteristics in previous depressed $v s$ healthy group comparison studies (eg, dichotomous diagnostic designations), increasing relevance of present findings to the extant literature. During a structured interview, clinicians established: the presence/absence of at least one comorbid anxiety diagnosis, the presence/absence of 'severe' depression, and the degree of recurrence of depressive episodes. Recurrent depression was dichotomized as $<3$ episodes $v s \geqslant 3$ episodes. Although this cut-point diverges from the clinical definition of 'recurrent depression' (2+ episodes), it has been previously linked to clinical outcomes and prognosis (Piet and Hougaard, 2011; Teasdale et al, 2000), and preserved an adequate distribution (eg, sufficient individuals in each cell) for analysis, while circumventing problems with distributional skewing. Gender and age were examined as biological/demographic factors with relevance in depression prevalence, presentation, and treatment 

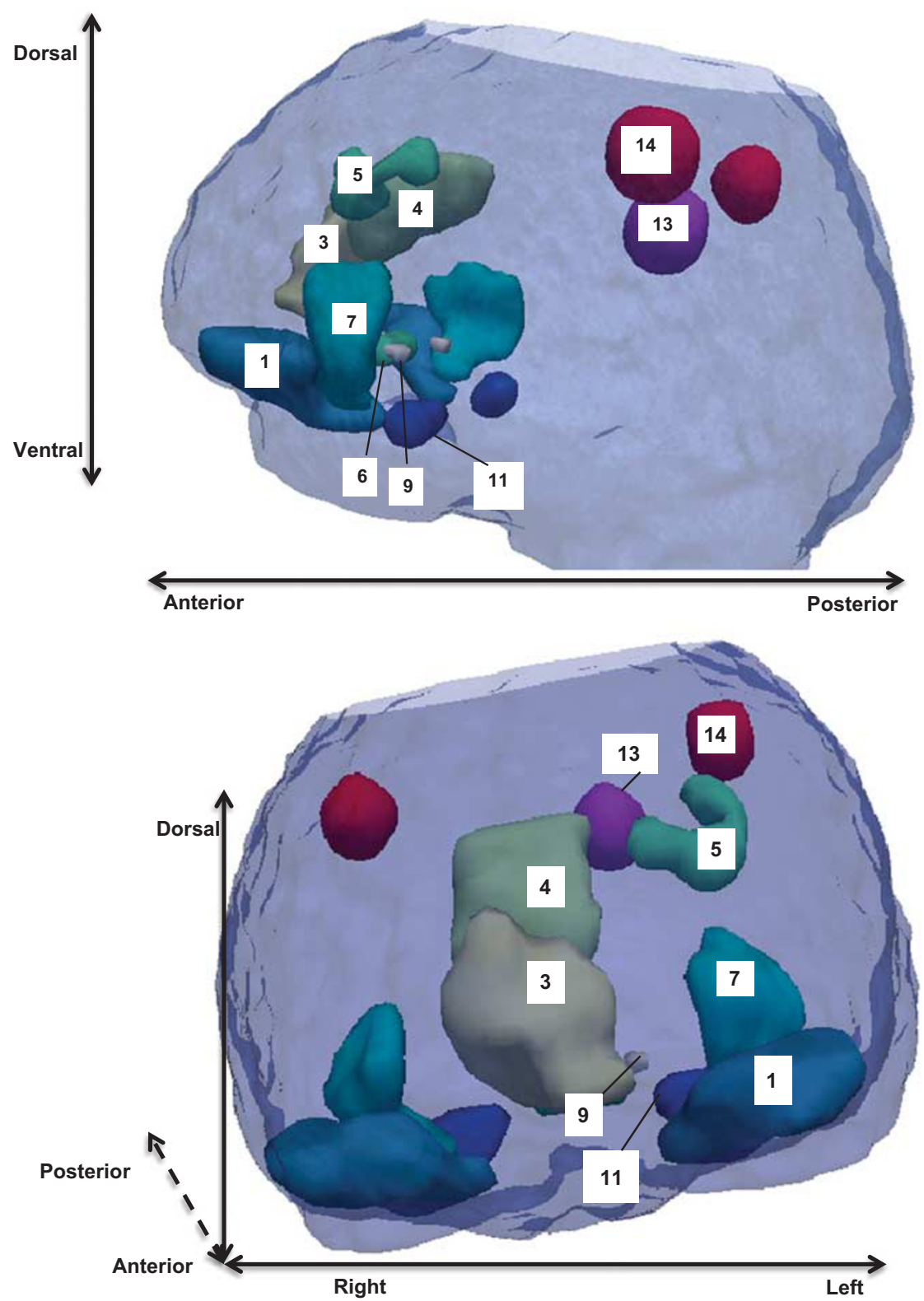

Figure I Three-dimensional renderings of region of interest (ROI) locations in template space. For ROls that are bilateral, a uniform color is used to label both hemispheres and regions are numerically labeled on the left hemisphere only; I = left ventrolateral PFC (L VLPFC); $2=R$ VLPFC; $3=$ perigenual anterior cingulate cortex (pgACC); $4=$ dorsal ACC (dACC); $5=L$ dorsolateral PFC ( $L$ DLPFC); $6=$ subgenual $A C C$ (sgACC); $7=L$ Insula; $8=R$ Insula; $9=L$ nucleus accumbens (NucAcc); 10=R NucAcc; II = L Amygdala; 12=R Amygdala; 13=posterior cingulate cortex (PCC); 14=left posterior parietal cortex (L Parietal); I $5=$ R Parietal. Figure reprinted from Biological Psychiatry, Vol 8I, Price et al, "Parsing Heterogeneity in the Brain Connectivity of Depressed and Healthy Adults During Positive Mood", p. 350, Copyright (2016), with permission from Elsevier.

(Cyranowski et al, 2000; Green et al, 2005; Szanto et al, 2003). Sources of missing data and overlap among external variables are discussed in the Supplementary Information.

\section{RESULTS}

\section{Connectivity Maps}

Group level. At the group level, connectivity paths depicted in Figure 2 were present, in addition to lagged autoregressive effects at every ROI. ROIs behaved as a strongly interconnected network, including numerous ipsilateral and within-network $\quad(\mathrm{VAN} \rightarrow \mathrm{VAN}$ and $\mathrm{CCN} \rightarrow \mathrm{CCN})$ connections, but notably lacked significant connections between the two DMN nodes.

Subgroups. Based on unsupervised search for the optimal number of subgroups, two subgroups emerged (see Supplementary Information for subgroup quality/stability information). Subgroup A contained 71\% $(n=57)$ of participants; hence, Subgroup B (29\% of participants; $n=23$ ) was considered to exhibit 'atypical' connectivity patterns relative to the majority of depressed patients. Subgroup was unrelated to the scanner where data were acquired $\left(\chi^{2}=0.93, p=0.335\right)$; to motion; and to other data quality measures (Supplementary 
a

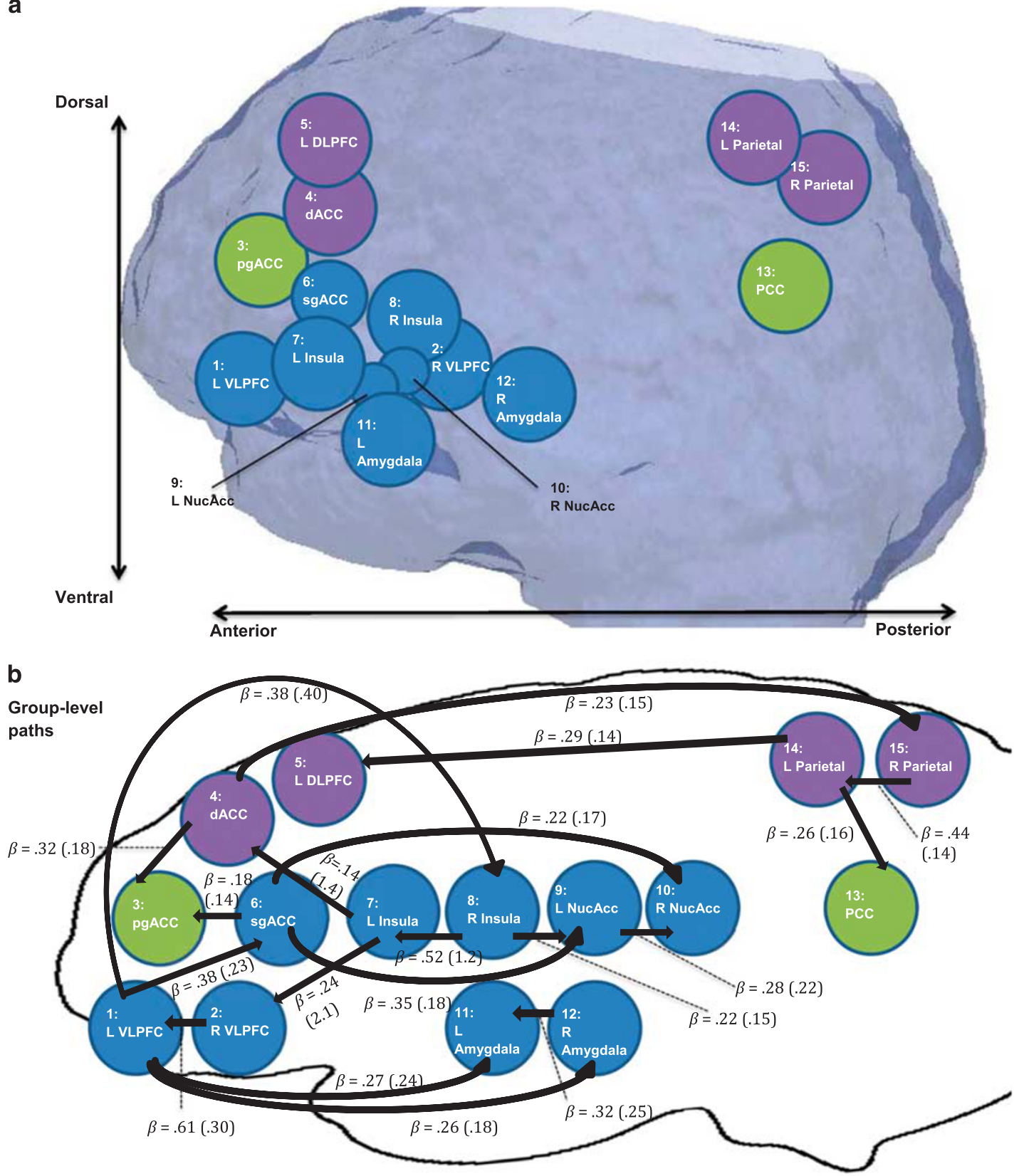

Figure 2 (a) Regions of interest (ROIs) represented as nodes in rough anatomical space. Nodes of the ventral affective network (VAN) are presented in blue; default mode network (DMN) in green; cognitive control network (CCN) in purple. (b) Group-level directed connectivity paths between regions of interest (flattened to two dimensions and stretched in space to facilitate visualization of all significant paths). All identified paths are contemporaneous with positive beta weights; additional lagged autoregressive (positive) paths were found for every ROI (not shown). Superimposed text displays beta-weights as mean (SD) across all individuals with a given path. See Supplementary Information for further discussion.

Information). In paths unique to each subgroup (Figure 3), only Subgroup A showed DMN $\rightarrow \mathrm{DMN}$ connectivity (a pgACC $\rightarrow$ PCC directed path). Subgroup A further exhibited a unique dACC $\rightarrow \mathrm{R}$ insula path, whereas Subgroup $B$ had a reversed direction of effect for dACC $\rightarrow$ L DLPFC, as well as an additional $\mathrm{dACC} \rightarrow \mathrm{R}$ parietal path and a $\mathrm{L}$ insula $\rightarrow R$ amygdala path.

\section{External Variables}

Clinical variables. Subgroup B was associated with multiple markers of poor prognosis in depression (Table 1). Comorbid anxiety was over-represented in Subgroup B (42.9,\%), a more than threefold increase in odds $\left(\chi^{2}=5.34, p=0.021\right)$. Highly recurrent depression was similarly over-represented in Subgroup B (63.2\%) relative to Subgroup A $\left(31.8 \% ; \chi^{2}=5.38\right.$, $p=0.020)$. Subgroup $\mathrm{B}$ had a nonsignificant overrepresentation of 'severe' depression diagnoses (38.1 vs 19.3\% in Subgroup A; $\chi^{2}=2.94, p=0.086$ ). See Supplementary Information for analyses of additional clinical variables.

Biological/demographic variables. Subgroup B was almost exclusively comprised of females $(87.0 \%, n=20 / 23)$ in 

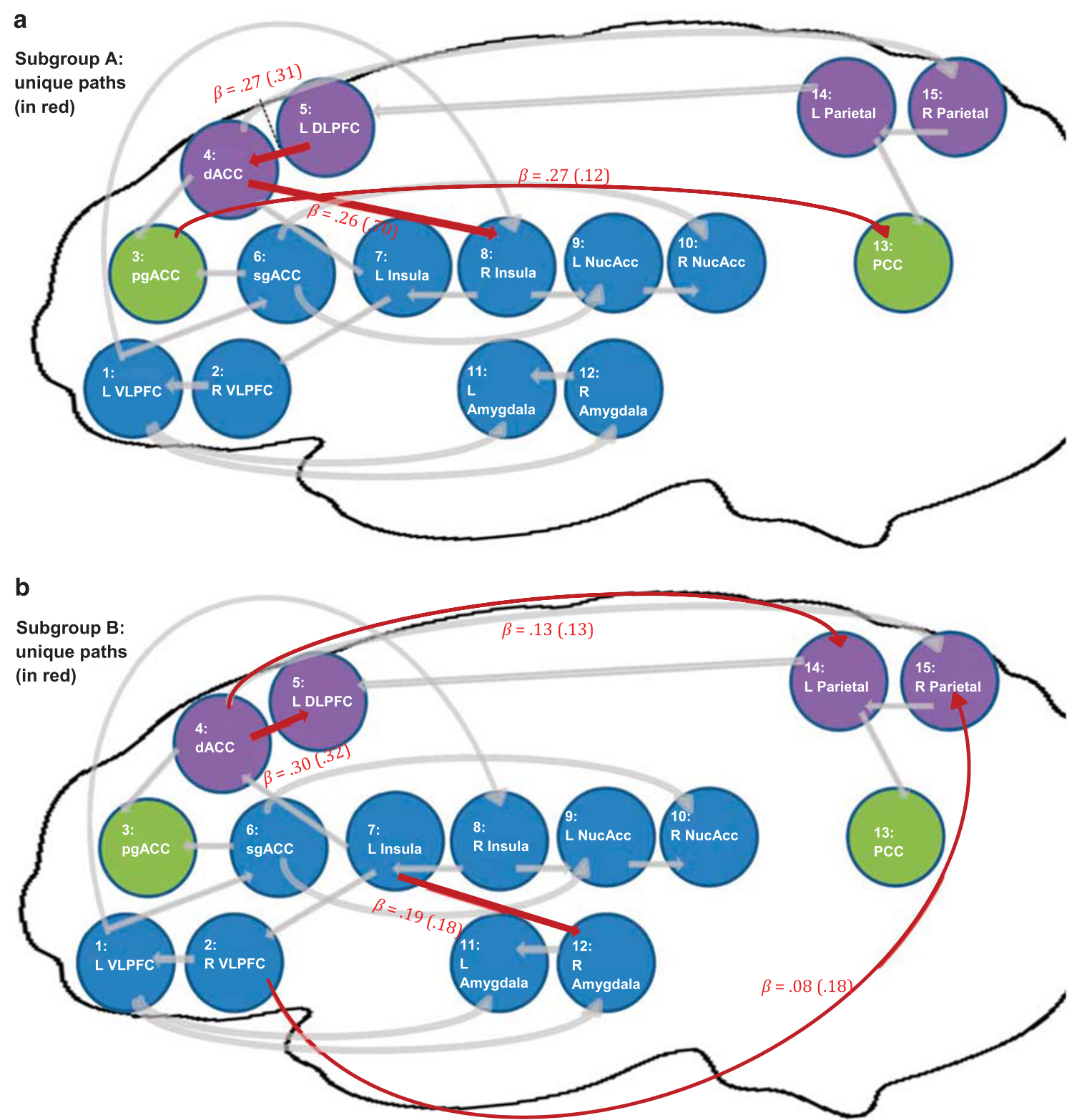

Figure 3 Subgroup-specific connectivity paths. All identified paths are contemporaneous with positive beta weights. Superimposed text displays betaweights as mean (SD) across all individuals with a given path. (a) Directed connectivity paths unique to subgroup A (in red), superimposed on group-level connectivity map (in gray). (b) Directed connectivity paths unique to subgroup B (in red), superimposed on group-level connectivity map (in gray).

contrast to Subgroup A $\left(64.9 \% ; \chi^{2}=3.89, p=0.049\right)$. Subgroups did not differ on age $\left(t_{78}=0.72, p=0.473\right)$.

Summary. In aggregate, analyses suggested connectivitybased subgroups had external clinical and real-world relevance with regard to key sample composition features.

\section{DISCUSSION}

In the present analysis, two data-driven RS connectivity subgroups were identified within a depressed sample using a robust method for recovering functional neural network structure among heterogeneous individuals. The larger group exhibited a directed pathway linking two hubs of the DMN, whereas a smaller subgroup showed an 'atypical' connectivity pattern where within-DMN connectivity was notably lacking and where dACC-driven paths were more prominent. Connectivity-based subgroups tracked with several clinical and demographic features, suggesting clinical relevance. The 'atypical' (smaller) subgroup was associated with markers previously linked to poor prognosis in depression, including comorbid anxiety and high episodic recurrence, and with female gender.

Strength of connectivity within the DMN is posited to reflect perseverative self-focus in the absence of exogenous instruction (Andrews-Hanna et al, 2010) and has been tied to negative rumination (Zhu et al, 2012), a perseverative form of cognition that increases risk for depression (NolenHoeksema et al, 1993). Our findings suggest there is a potent direct influence from the pgACC to the PCC-two prominent nodes of the DMN-in the majority, but not all, of depressed patients. A minority of patients lack this specific 
path after controlling for the influence of all other regions included in our network, suggesting the functional influence of these two regions on one another is diminished in this subgroup, with fluctuations in activity over time being more completely explained by other inputs present across the entire sample (depicted in Figure 2b). Meta-analytic findings suggest that, on average, depression is associated with hyperconnectivity between PCC and medial PFC regions (Kaiser et al, 2015). Although caution is warranted given the novel directed connectivity method applied here, current findings could suggest such patterns are driven by the majority of depressed patients, whereas a sizable minority do not display strong connectivity within this circuit. Differences in sampling of these two depressive subgroups across studies might therefore yield differential strength of findings which, based on our observations for external variables, may be reflected in clinical and demographic features. Because of our focus on external variables representing widely reported sample characteristics, this hypothesis could be readily tested in future meta-analyses making use of the large number of existing non-directed (correlational) connectivity studies. Within-subject variability in connectivity patterns (ie, coupling dynamics) (Hutchison and Morton, 2015) may also contribute to mixed findings. Quantifying such dynamics, ideally in conjunction with an approach capable of parsing between-subject heterogeneity, represents a promising novel direction for patient classification (Rashid et al, 2016).

Our findings were dictated by differential patterns present in the neural connectivity data itself, rather than on a priori selection of individual differences variables. Nevertheless, findings are potentially informative regarding the neural substrates of key sources of depressive heterogeneity observed clinically. Anxious depression is a widely-recognized clinical subgroup associated with poor prognosis (Fava et al, 2004, 2008), which has distinct neural features including altered activity and connectivity within the networks studied here (Demenescu et al, 2011; Etkin and Schatzberg, 2011b; Oathes et al, 2015; Pannekoek et al, 2015; van Tol et al, 2012; van Tol et al, 2011). Our findings are potentially consistent with prior studies suggesting that both common (here, group-level) and unique (subgroup-specific) neural substrates underlie depression and anxiety. The data-driven approach reveals that connectivity features track with diagnostic boundaries, yet do not overlap perfectly with these features, suggesting connectivity patterns contain unique information that could be obscured by conventional group comparisons.

One network distinction apparent across the two subgroups included a directed path from left DLPFC $\rightarrow$ dACC in Subgroup A, which was reversed in Subgroup B. Subgroup B additionally showed a unique dACC $\rightarrow$ parietal path, whereas Subgroup A showed a unique dACC $\rightarrow$ insula path. Although the dACC was historically construed as a strictly 'cold' cognitive region (Bush et al, 2000), contemporary conceptualizations highlight its additional role in the generation and expression of negative emotion (Etkin et al, 2011a), in particular within the context of anxiety research. Thus, its pivotal role in defining depression subgroups that tracked with comorbid anxiety may be significant. For example, greater overall dACC-driven activity might reflect a greater influence of fear-driven processing over cognition. Two additional specific paths present in Subgroup B, right VLPFC $\rightarrow$ right parietal and left insula $\rightarrow$ right amygdala, could reflect additional VAN-driven processing at rest. This is potentially consistent with a previous report of increased RS connectivity between VAN and CCN regions that was specific to comorbid anxiety and depression (Pannekoek et al, 2015) and also with the prominent roles for exaggerated 'bottomup' influences of the VAN in neuroanatomical models of both anxiety and depression (Drevets et al, 2008; Etkin et al, 2009; Mathew et al, 2008; Price and Drevets, 2010). Notably, in supplemental analyses, connectivity-based subgroups did not track primarily with any one specific anxiety diagnosis present in the current sample, nor with a continuous measure of anxiety, suggesting the present finding related only to transdiagnostic, clinically diagnosed anxiety. Given that anxiety is itself a highly heterogeneous condition, future studies should include larger and more diverse anxiety presentations, with further attention to heterogeneity across both categorical and continuous measures.

At least $50-60 \%$ of depressed patients experience more than one episode (American et al, 2001) and it is wellestablished that risk of future depressive episodes increases with each prior episode (Solomon et al, 2000), becoming particularly $(\geqslant 90 \%)$ likely in individuals who have had three or more episodes (American et al, 2001). This pattern could reflect increasingly well-practiced negative cognitions (Beck and Bredemeier, 2016) that become easily re-activated following subsequent stressors through a process termed the 'kindling effect' (Monroe and Harkness, 2005; Post, 1992). Alternatively, there may be two subgroups of patients, each with 'stable-liability' - one prone to high recurrence and another who will experience only a few sporadic (if any) additional episodes (Anderson et al, 2016). Few studies in human patients have examined the neural substrates of differential recurrence. In at least some patients with a history of high recurrence, our data suggest involvement of a functional neural architecture with stronger baseline influences stemming from VAN regions and from dACC to other $\mathrm{CCN}$ regions, which could be consistent with stronger internally driven affective 'schema' that co-opt cognitive resources. However, findings simultaneously highlight individual differences in connectivity, with a full half of patients with high recurrence showing greater similarity to the 'typical' (larger) depressed subgroup. This could indicate more than one neurocognitive pathway to recurrence, only one of which was characterized here; for example, patients in Subgroup A with high recurrence might differ in nonassessed neural networks or during distinct task states (eg, under emotional provocation). Likewise, $37 \%$ of patients in Subgroup B did not report high recurrence at the time of assessment. With prospective follow-up, a highly recurrent pattern might become evident in these specific patients-a testable hypothesis for future work.

One other sample characteristic-gender-tracked with connectivity subgroup, such that the 'atypical' subgroup was almost exclusively female. Females are $2 \times$ more likely than males to experience MDD, a gender discrepancy that first appears in adolescence. Posited mechanisms of this gender gap include biological and psychosocial factors (Cyranowski et al, 2000; Angold and Costello, 2006; Crone and Dahl, 2012; Hyde et al, 2008). Depressed females also show distinct clinical features, including higher rates of comorbid anxiety (Schuch et al, 2014). In RS data, healthy females have shown greater connectivity of amygdala subregions to numerous 
VAN and CCN regions (Engman et al, 2016; Kogler et al, 2016; Lopez-Larson et al, 2011), possibly consistent with the unique insula $\rightarrow$ amygdalar pathway found here in Subgroup B. However, group comparisons by gender may set up a false dichotomy, as females were over-represented in Subgroup B, but also well-represented (65\%) in Subgroup A. Future analyses should aim to delineate additional biobehavioral features that may distinguish those depressed women in the female-heavy subgroup from those biologically classified together with the vast majority of depressed men.

Using identical S-GIMME methods applied to data collected during a positive mood induction, we previously reported in an overlapping sample (which also included healthy controls) that the majority of depressed patients showed widespread hyperconnectivity across this network of regions, in particular for ventrally driven pathways (Price et al, 2017). Here we explicitly searched for and parsed heterogeneity within depressed patients at rest. The subgrouping patterns from the two analyses did not appear highly convergent either in terms of the distinguishing (subgroup-specific) directed pathways or the specific parsing of patients (Supplementary Information), suggesting the two approaches yielded unique information that could be jointly informative for practical goals such as predicting clinical trajectories. Data-driven subgroups derived from correlational (ie, non-directional) patterns within depressed patients' RS data were also divergent anatomically from those identified here, although they likewise tracked with anxious phenotypes of depression (Drysdale et al, 2017). Future work should empirically establish combinations of information that are most informative for clinically imperative tasks (eg, matching patients to specific interventions).

\section{Limitations}

Although regions analyzed in the network were limited to 15 to increase interpretability and reduce processing time, results may have varied with the inclusion of different or additional regions, as many potentially relevant regions (eg, dorsomedial PFC beyond the ACC boundaries) were omitted in favor of the present set. Alternate forms of data-driven subtype analysis, recently applied to RS connectivity in depression (Drysdale et al, 2017), are able to consider a wider network of brain regions, although these methods differ in that they rely on correlational patterns rather than characterizing directional influences-a method that may be less accurate for subgrouping according to simulations (Gates et $a l$, in press). Data-driven subgroups are dictated by the individuals in the sample and may have differed with a larger sample or greater representation of certain groups (eg, older participants and more ethnic minorities). Replication is essential to understand the robustness and generalizability of these specific subgroups. In addition, the hard-clustering approach did not allow for multiple subgroup membership, meaning that some individuals could be similar to both subgroups. External clinical variables were assessed crosssectionally; testing prospective prediction and the stability of subgroups over time are critical next steps. Although assessment tools that accurately parse biobehavioral heterogeneity may one day be informative in clinical decision making, numerous hurdles remain, including: external validation of subgroups in larger, independent samples; developing prediction algorithms with sufficiently high accuracy for specific outcomes; and ideally, translation to clinic-ready assessment methods, given that fMRI is expensive and not widely available to patients. Finally, the directional brain pathways quantified here based on temporal patterns require external validation, eg, using brain stimulation methods to experimentally manipulate one region and observe downstream effects on other regions.

\section{CONCLUSIONS}

RS connectivity, an index of coordinated brain activation across a neural network, is posited to reflect stable neural functional architecture impacting the brain's reactive responses to a wide range of stimuli. Although RS connectivity alterations are widely implicated in depression, our findings using a robust data-driven algorithm suggest no one-size-fits-all pattern. In particular, the lack of reliable DMN connectivity within $29 \%$ of patients suggests a replicated biomarker of depression, DMN hyperconnectivity, may not apply equivalently to all patients. A clinical implication is that diverse depression treatments that appear to normalize DMN hyperconnectivity (eg, conventional antidepressants, intravenous ketamine, and mindfulness meditation; (Berkovich-Ohana et al, 2016; Karim et al, 2016; Lv et al, 2016), as well as targeted mechanistic treatments (eg, neurofeedback to decrease DMN connectivity (Zhang et al, 2013) and transcranial magnetic stimulation (Drysdale et al, 2017)), could be ill-matched for a sizable minority of patients. These patients might benefit instead from treatments that reduce dACC- and VAN-driven influenceswhich could represent fear-driven cognitive processing, particularly given the link observed to comorbid anxiety. Identifying treatments capable of addressing the atypical connectivity pattern may be particularly clinically impactful, as this pattern tracked with markers of poor prognosis.

\section{FUNDING AND DISCLOSURE}

This research was supported by grants R21EB015573 (NIBIB; Gates), MH074807, MH082998, MH58356, MH58397 and MH69618 from the National Institutes of Health, by the Pittsburgh Foundation, and by the Emmerling Fund. Dr Price is supported by NIH Career Development Award K23MH100259. Dr. Gates' work is supported by the NIH. Mr. Kraynak reports no potential conflicts of interest. Dr. Thase has financial relationships with the following commercial entities: Alkermes, Shire US Inc., Allergan, AstraZeneca, Bristol-Myers Squibb Company, Cerecor, Inc., Eli Lilly \& Co., Forest Laboratories, Gerson Lehrman Group, FabreKramer Pharmaceuticals, Inc., Glaxo-SmithKline, Guidepoint Global, H. Lundbeck A/S, MedAvante, Inc., Merck and Co. Inc., Moksha8, Naurex, Inc., Neuronetics, Inc., Novartis, Ortho-McNeil Pharmaceuticals, Otsuka, Pamlab, LLC, Pfizer, Sunovion Pharmaceuticals, Inc., Trius Therapeutical, Inc., and Takeda. Dr. Siegle's work is supported by the NIH.

\section{ACKNOWLEDGMENTS}

We gratefully acknowledge Stephanie Lane for her contributions to the present analyses. 


\section{REFERENCES}

American Psychiatric Association (2001). Diagnostic and Statistical Manual of Mental Disorders-Text Revision4 ednAPA: Washington, DC.

Anderson SF, Monroe SM, Rohde P, Lewinsohn PM (2016). Questioning kindling: an analysis of cycle acceleration in unipolar depression. Clin Psychol Sci 4: 229-238.

Andrews-Hanna JR, Reidler JS, Sepulcre J, Poulin R, Buckner RL (2010). Functional-anatomic fractionation of the brain's default network. Neuron 65: 550-562.

Angold A, Costello EJ (2006). Puberty and depression. Child Adolesc Psychiatr Clin N Am 15: 919-937.

Beck AT, Bredemeier K (2016). A unified model of depression: integrating clinical, cognitive, biological, and evolutionary perspectives. Clin Psychol Science 4: 596-619.

Berkovich-Ohana A, Harel M, Hahamy A, Arieli A, Malach R (2016). Data for default network reduced functional connectivity in meditators, negatively correlated with meditation expertise. Data Brief 8: 910-914.

Bush G, Luu P, Posner MI (2000). Cognitive and emotional influences in anterior cingulate cortex. Trends Cogn Sci 4: 215-222.

Clementz BA, Sweeney JA, Hamm JP, Ivleva EI, Ethridge LE, Pearlson GD et al (2016). Identification of distinct psychosis biotypes using brain-based biomarkers. Am J Psychiatry 173: 373-384.

Crone EA, Dahl RE (2012). Understanding adolescence as a period of social-affective engagement and goal flexibility. Nat Rev Neurosci 13: 636-650.

Cyranowski JM, Frank E, Young E, Shear MK (2000). Adolescent onset of the gender difference in lifetime rates of major depression: a theoretical model. Arch Gen Psychiatry 57: 21-27.

Demenescu LR, Renken R, Kortekaas R, van Tol MJ, Marsman JB, van Buchem MA et al (2011). Neural correlates of perception of emotional facial expressions in out-patients with mild-tomoderate depression and anxiety. A multicenter fMRI study. Psychol Med 41: 2253-2264.

Drevets WC, Price JL, Furey ML (2008). Brain structural and functional abnormalities in mood disorders: implications for neurocircuitry models of depression. Brain Struct Funct 213: 93-118.

Drysdale AT, Grosenick L, Downar J, Dunlop K, Mansouri F, Meng Y et al (2017). Resting-state connectivity biomarkers define neurophysiological subtypes of depression. Nat Med 23: 28-38.

Engman J, Linnman C, Van Dijk KR, Milad MR (2016). Amygdala subnuclei resting-state functional connectivity sex and estrogen differences. Psychoneuroendocrinology 63: 34-42.

Etkin A, Egner T, Kalisch R (2011a). Emotional processing in anterior cingulate and medial prefrontal cortex. Trends Cogn Sci 15: 85-93.

Etkin A, Prater KE, Schatzberg AF, Menon V, Greicius MD (2009). Disrupted amygdalar subregion functional connectivity and evidence of a compensatory network in generalized anxiety disorder. Arch Gen Psychiatry 66: 1361-1372.

Etkin A, Schatzberg AF (2011b). Common abnormalities and disorder-specific compensation during implicit regulation of emotional processing in generalized anxiety and major depressive disorders. Am J Psychiatry 168: 968-978.

Fava M, Alpert JE, Carmin CN, Wisniewski SR, Trivedi MH, Biggs MM et al (2004). Clinical correlates and symptom patterns of anxious depression among patients with major depressive disorder in STAR ${ }^{\star}$ D. Psychol Med 34: 1299-1308.

Fava M, Rush AJ, Alpert JE, Balasubramani GK, Wisniewski SR, Carmin CN et al (2008). Difference in treatment outcome in outpatients with anxious versus nonanxious depression: a STAR $^{\star}$ D report. Am J Psychiatry 165: 342-351.

Finn ES, Shen X, Scheinost D, Rosenberg MD, Huang J, Chun MM et al (2015). Functional connectome fingerprinting: identifying individuals using patterns of brain connectivity. Nat Neurosci 18: $1664-1671$.

Fried EI, Nesse RM (2014). Depression is not a consistent syndrome: an investigation of unique symptom patterns in the STAR ${ }^{\star}$ D study. J Affect Disord 172C: 96-102.

Gates KM, Lane ST, Varangas E, Giovanello K, Guiskewicz K (2017). Unsupervised classification during time series model selection. Multivariate Behav Res 52: 129-148.

Gates KM, Molenaar PC (2012). Group search algorithm recovers effective connectivity maps for individuals in homogeneous and heterogeneous samples. Neuroimage 63: 310-319.

Green H, McGinnity Á, Meltzer H, Ford T, Goodman R (2005). Mental Health of Children and Young People in Great Britain, 2004. Palgrave Macmillan: Basingstoke.

Hasler G, Northoff G (2011). Discovering imaging endophenotypes for major depression. Mol Psychiatry 16: 604-619.

Heller AS, Johnstone T, Shackman AJ, Light SN, Peterson MJ, Kolden GG et al (2009). Reduced capacity to sustain positive emotion in major depression reflects diminished maintenance of fronto-striatal brain activation. Proc Natl Acad Sci USA 106: 22445-22450.

Hutchison RM, Morton JB (2015). Tracking the brain's functional coupling dynamics over development. J Neurosci 35: 6849-6859.

Hyde JS, Mezulis AH, Abramson LY (2008). The ABCs of depression: integrating affective, biological, and cognitive models to explain the emergence of the gender difference in depression. Psychol Rev 115: 291.

Kaiser RH, Andrews-Hanna JR, Wager TD, Pizzagalli DA (2015). Large-scale network dysfunction in major depressive disorder: a meta-analysis of resting-state functional connectivity. JAMA Psychiatry 72: 603-611.

Karalunas SL, Fair D, Musser ED, Aykes K, Iyer SP, Nigg JT (2014). Subtyping attention-deficit/hyperactivity disorder using temperament dimensions: toward biologically based nosologic criteria. JAMA Psychiatry 71: 1015-1024.

Karim HT, Andreescu C, Tudorascu D, Smagula SF, Butters MA, Karp JF et al (2016). Intrinsic functional connectivity in late-life depression: trajectories over the course of pharmacotherapy in remitters and non-remitters. Mol Psychiatry 22: 450-457.

Kim J, Zhu W, Chang L, Bentler PM, Ernst T (2007). Unified structural equation modeling approach for the analysis of multisubject, multivariate functional MRI data. Hum Brain Mapp 28: 85-93.

Kogler L, Muller VI, Seidel EM, Boubela R, Kalcher K, Moser E et al (2016). Sex differences in the functional connectivity of the amygdalae in association with cortisol. NeuroImage 134: 410-423.

Lane ST, Gates KM, Molenaar PCM (2015). gimme https://cran.rproject.org/web/packages/gimme/index.html.

Lopez-Larson MP, Anderson JS, Ferguson MA, Yurgelun-Todd D (2011). Local brain connectivity and associations with gender and age. Dev Cogn Neurosci 1: 187-197.

Lv Q, Yang L, Li G, Wang Z, Shen Z, Yu W et al (2016). Large-scale persistent network reconfiguration induced by ketamine in anesthetized monkeys: relevance to mood disorders. Biol Psychiatry 79: 765-775.

Mathew SJ, Price RB, Charney DS (2008). Recent advances in the neurobiology of anxiety disorders: implications for novel therapeutics. Am J Med Genet C Semin Med Genet 148C: 89-98.

Miller MB, Van Horn JD, Wolford GL, Handy TC, Valsangkar-Smyth M, Inati S et al (2002). Extensive individual differences in brain activations associated with episodic retrieval are reliable over time. J Cogn Neurosci 14: 1200-1214.

Molenaar PC, Campbell CG (2009). The new person-specific paradigm in psychology. Curr Directions Psychol 18: 112-117.

Monroe SM, Harkness KL (2005). Life stress, the "kindling" hypothesis, and the recurrence of depression: considerations from a life stress perspective. Psychol Rev 112: 417-445. 
Mumford JA, Ramsey JD (2014). Bayesian networks for fMRI: a primer. Neuroimage 86: 573-582.

Nolen-Hoeksema S, Morrow J, Fredrickson BL (1993). Response styles and the duration of episodes of depressed mood. J Abnorm Psychol 102: 20-28.

Oathes DJ, Patenaude B, Schatzberg AF, Etkin A (2015). Neurobiological signatures of anxiety and depression in resting-state functional magnetic resonance imaging. Biol Psychiatry 77: 385-393.

Orman G, Labatut V (2009). A comparison of community detection algorithms on artificial networks. In: Gama J, Costa VS, Jorge AM, Brazdil PB (eds). Discovery Science. Springer Berline Heidelberg: Berlin, Heidelberg. pp 242-256.

Pannekoek JN, van der Werff SJ, van Tol MJ, Veltman DJ, Aleman A, Zitman FG et al (2015). Investigating distinct and common abnormalities of resting-state functional connectivity in depression, anxiety, and their comorbid states. Eur Neuropsychopharmacol 25: 1933-1942.

Piet J, Hougaard E (2011). The effect of mindfulness-based cognitive therapy for prevention of relapse in recurrent major depressive disorder: a systematic review and meta-analysis. Clin Psychol Rev 31: 1032-1040.

Pons P, Latapy M (2006). Computing communities in large networks using random walks. J Graph Algorithms Applications 10: 191-218.

Post RM (1992). Transduction of psychosocial stress into the neurobiology of recurrent affective disorder. Am J Psychiatry 149: 999-1010.

Price JL, Drevets WC (2010). Neurocircuitry of mood disorders. Neuropsychopharmacology 35: 192-216.

Price RB, Lane S, Gates KM, Kraynak TE, Horner MS, Thase ME et al (2017). Parsing heterogeneity in directed brain connectivity during positive mood: A community detection analysis in depressed and healthy adults. Biol Psychiatry 81: 347-357.

Rashid B, Arbabshirani MR, Damaraju E, Cetin MS, Miller R, Pearlson GD et al (2016). Classification of schizophrenia and bipolar patients using static and dynamic resting-state fMRI brain connectivity. NeuroImage 134: 645-657.

Schuch JJ, Roest AM, Nolen WA, Penninx BW, de Jonge P (2014). Gender differences in major depressive disorder: results from the Netherlands study of depression and anxiety. J Affect Disord 156: 156-163.
Siegle GJ, Thompson WK, Collier A, Berman SR, Feldmiller J, Thase ME et al (2012). Towards clinically useful neuroimaging in depression treatment: Is subgenual cingulate activity robustly prognostic for depression outcome in Cognitive Therapy across studies, scanners, and patient characteristics? Arch Gen Psychiatry 69: 913-924.

Smith SM, Miller KL, Salimi-Khorshidi G, Webster M, Beckmann CF, Nichols TE et al (2011). Network modelling methods for FMRI. Neuroimage 54: 875-891.

Solomon DA, Keller MB, Leon AC, Mueller TI, Lavori PW, Shea MT et al (2000). Multiple recurrences of major depressive disorder. Am J Psychiatry 157: 229-233.

Sporns O, Chialvo DR, Kaiser M, Hilgetag CC (2004). Organization, development and function of complex brain networks. Trends Cogn Sci 8: 418-425.

Szanto K, Mulsant BH, Houck P, Dew MA, Reynolds CF 3rd (2003). Occurrence and course of suicidality during short-term treatment of late-life depression. Arch Gen Psychiatry 60: 610-617.

Teasdale JD, Segal ZV, Williams JMG, Ridgeway VA, Soulsby JM, Lau MA (2000). Prevention of relapse/recurrence in major depression by mindfulness-based cognitive therapy. J Consult Clin Psychol 68: 615-623.

van Tol M-J, Demenescu LR, van der Wee NJa, Kortekaas R, Marjan MAN, Boer JaD et al (2012). Functional magnetic resonance imaging correlates of emotional word encoding and recognition in depression and anxiety disorders. Biol Psychiatry 71: 593-602.

van Tol MJ, van der Wee NJ, Demenescu LR, Nielen MM, Aleman A, Renken R et al (2011). Functional MRI correlates of visuospatial planning in out-patient depression and anxiety. Acta Psychiatr Scand 124: 273-284.

Yang Z, Xu Y, Xu T, Hoy CW, Handwerker DA, Chen G et al (2014). Brain network informed subject community detection in early-onset schizophrenia. Sci Rep 4: 5549.

Zhang G, Zhang H, Li X, Zhao X, Yao L, Long Z (2013). Functional alteration of the DMN by learned regulation of the PCC using real-time fMRI. IEEE Trans Neural Syst Rehabil Eng 21: 595-606.

Zhu X, Wang X, Xiao J, Liao J, Zhong M, Wang W et al (2012). Evidence of a dissociation pattern in resting-state default mode network connectivity in first-episode, treatment-naive major depression patients. Biol Psychiatry 71: 611-617.

Supplementary Information accompanies the paper on the Neuropsychopharmacology website (http://www.nature.com/npp) 